\title{
AVALIAÇÃO E VIABILIDADE DE IMPRESSORAS 3D DE BAIXO CUSTO PARA REALIZAÇÃO DE ATIVIDADES ACADÊMICAS
}

\author{
Carlos Maurício Sacchelli-carlos.sacchelli@gmail.com \\ Guilherme Cervi Garcia-guilhermecervigarcia@gmail.com \\ Thiago Almeida Eidt - thiagoeidt@hotmail.com \\ Universidade Federal de Santa Catarina - Campus Joinville \\ Rua Dona Francisca, 8300, Zona Industrial Norte, Bloco U \\ 89219-600 - Joinville - Santa Catarina
}

\begin{abstract}
Resumo: Este trabalho foi realizado por membros do Programa de Educação Tutorial das Engenharias da Mobilidade (PET EMB) do campus de Joinville da Universidade Federal de Santa Catarina (UFSC). Trata-se de um relato de experiência que tem como objetivo analisar diferentes projetos de manufatura aditiva e também descrever as experiências dos membros na construção de um protótipo de projeto aberto de baixo custo de um equipamento de impressão pelo processo FDM - Modelagem de Depósito Fundido. Durante a construção do projeto foram encontrados desafios, dificuldades e aprendizagens, constituindo em uma experiência que contribuiu na formação dos membros envolvidos.
\end{abstract}

Palavras-chave: Manufatura aditiva. Projetos abertos. FDM. Baixo custo. PET.

\section{INTRODUÇÃO}

A palavra manufatura, derivada do latim medieval, manu, mão, e factura, feitio, pressupõe o significado de fabricação por métodos não naturais, ou seja, pela interferência racional ou física do Homem. Em sua definição de lean manufacturing, Shah e Ward (2003) apresentam o termo como um método de "aumento de produtividade, isto é, fazer mais com menos recursos e suprir fontes de desperdícios ao longo da cadeia de valor" (JABBOUR et al., 2013). Pode-se deduzir que manufatura, compreende todos os processos de produção e transformação com um fim útil e lucrativo.

Redwood, Schöffer e Garret (2017) distinguem a manufatura em três vertentes: manufatura de formas, subtrativa e aditiva. A Manufatura Aditiva - MA, popularmente chamada de impressão 3D, é o processo de construção por camadas, sendo processada uma camada por vez. A MA é adequada para baixos volumes, geometrias complexas, incapazes de serem produzidas pelos outros tipos de processos de fabricação ou quando uma peça única é requerida. 
O primeiro artigo sobre a manufatura aditiva foi desenvolvido por um pesquisador japonês, Hideo Kodama, em 1981, intitulado "Métodos automáticos para a fabricação de modelos plásticos tridimensionais com polímeros de foto-endurecimento" onde foi discutida a solidificação em camadas de foto polímeros. Posteriormente, em 1984, Charles Chuck Hull criou a primeira patente de uma impressora 3D, sendo que esta utilizava o método SLA, Stereolithography (Estereolitografia). Já em 1989, Scott Crump, criou o processo FDM - Fused Deposition Modelling (Modelagem de Depósito Fundido) tornando-se o tipo de impressão mais popular (PAOLETTI e CECCON, 2018).

O método FDM apresenta diversas vantagens tais como: os custos (de aquisição, operação e material) são menores em relação aos demais métodos, menor desperdício de material, baixa necessidade de limpeza da plataforma e exige uma menor necessidade de espaço (RAULINO, 2011). O processo FDM é uma técnica de modelagem por extrusão de termoplástico, na qual o material passa por um bico extrusor aquecido, que por sua vez é depositado em uma plataforma. Assim que a máquina termina a primeira camada, a plataforma se desloca no eixo vertical para possibilitar a deposição de outra, essas etapas são repetidas até o objeto formar-se. Este processo é indicado para ser usado em ambientes acadêmicos, uso interno de empresas e para produtos customizáveis.

De maneira geral os equipamentos destinados a MA são de custos elevados, o que dificulta o seu uso por uma quantidade maior de empresas, contudo nos últimos anos, algumas destas tecnologias tiveram o seu domínio tornado público, isto quer dizer que, qualquer empresa poderia fabricar equipamentos que se destinam a realizar certos processos de MA.

Nos últimos anos com a internet atingindo um número maior de pessoas, se observou que vários projetos abertos de máquinas de MA foram disponibilizados para que as pessoas que tivessem interesse conseguissem construir e utilizar estes equipamentos com um custo mais baixo.

Este artigo foi proposto, tendo como objetivo principal analisar alguns projetos abertos disponíveis, bem como realizar a sua construção. Para isso o texto está organizado em três seções onde são abordados os tipos de processos, o que são projetos abertos e uma breve explicação de como foi montado a máquina de MA.

\section{TIPOS DE PROCESSOS}

Volpato et al. (2007) expõe e descreve quatro principais processos de impressão 3D: Sinterização a Laser - SLS, FDM, SLA e a Fabricação de Objetos Laminados. Um dos objetivos da MA é fornecer uma peça funcional sem processos e materiais intermediários. O primeiro método citado é um dos poucos que possuem a capacidade de produzir peças sólidas de metal e cerâmica (AGARWALA et al., 1995). O que sinteriza o material em forma de pó é um laser de $\mathrm{CO}_{2}$. Tal feixe de laser passa por este material sinterizando as partículas e formando uma camada (SILVA, 2019).

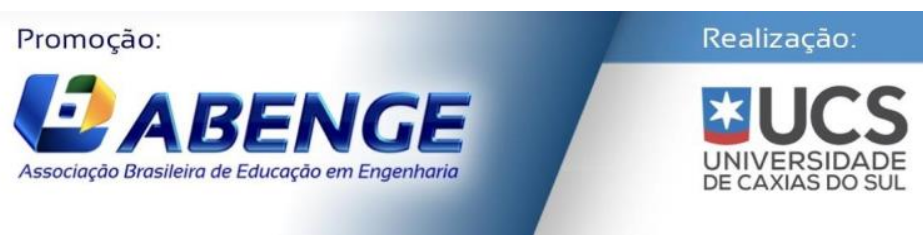


Em seu artigo sobre Modelagem por Fusão e Deposição, Masood (1996) define esta técnica como um processo pelos quais objetos físicos são criados diretamente de um design elaborado por um software CAD, depositando material extrudado camada por camada. Evans (2012) e Silva (2019) ressaltam que o material extrudado normalmente é um polímero na forma de filamentos e que o processo de fabricação ocorre pela sobreposição das camadas dos filamentos seguindo os contornos do modelo digital proposto. O FDM (Figura 1) integra várias áreas, são elas: design auxiliado por computador (CAD), ciência dos polímeros, controle número computadorizado e tecnologia de extrusão.

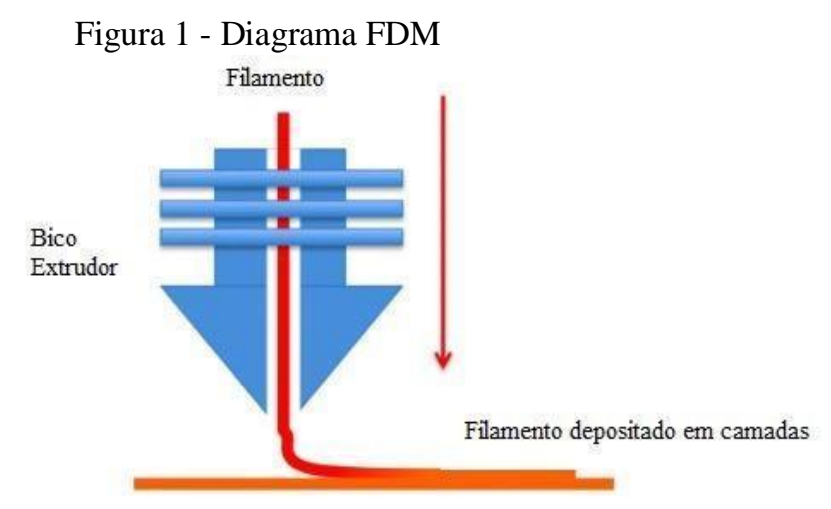

Fonte: Grames E. ${ }^{1}$

A estereolitografia - SLA é um conceito de manufatura baseada na repetição de duas diferentes etapas: solidificação de uma camada do produto, e o revestimento ou cobertura do topo do produto parcialmente construído com uma nova camada de resina líquida (RENAP e KRUTH, 1995).

Por fim, Volpato et al. (2007) e Silva (2019) comentam que a fabricação por objetos laminados se baseia na deposição de folhas de materiais contendo uma substância adesiva em uma de suas faces. Entre a reposição de tais camadas ocorre um aquecimento para a ativação do adesivo. Um laser no dela a camada atual de acordo com o perfil da peça e o ciclo se repete até que se tenha a geometria completa da peça.

Dentre estas tecnologias, a mais utilizada hoje é o FDM, pois, comparada à outros métodos possuem uma alta capacidade de fabricar componentes usando o mínimo de energia e matériaprima.

Com o domínio público do método FDM, uma grande comunidade de desenvolvedores começou a usar a tecnologia que posteriormente foi renomeada para FFF - Fused Filament Fabrication (Fabricação de Filamento Fundido), para não haver conflitos.

\footnotetext{
${ }^{1}$ Gráfico retirado de https://all3dp.com/2/fused-deposition-modeling-fdm-3d-printing-simply-explained/
}

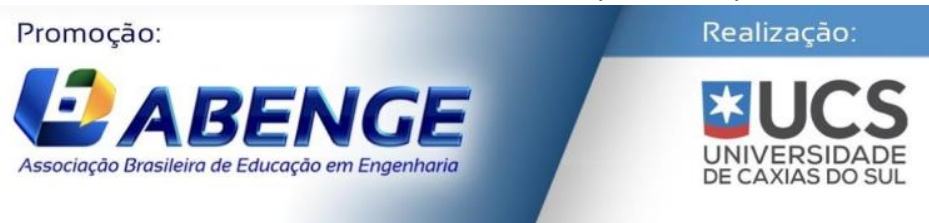


A tecnologia é de baixo custo por algumas características pontuais, Sendo elas: a popularidade a partir de 1992, o material do filamento usado é mais barato, a impressora requer peças de baixo custo sendo que a mesma pode fabricar algumas delas e os projetos envolvidos são mais baratos.

Os projetos utilizando FDM são mais baratos pela impressão ser de baixa precisão comparada a outros métodos como SLA e SLS, e também porque essas tecnologias de impressoras custam por unidade de área e de volume do projeto, e comumente isso não acontece no FDM (PANNETT, 2019) (GRAMES, 2019).

\section{PROJETOS ABERTOS}

Os projetos abertos possuem acesso universal e podem ser redistribuídos ou subsequentemente melhorados de acordo com o interesse dos usuários (STEENBEEK et al., 2015), apesar de ainda não serem amplamente conhecidos, estão conquistando mais espaço no mercado. Iniciativas como a RepRap e o Github são cada vez mais conhecidas e consequentemente fomentam a inovação e ampliam o acesso à diversas novas ideias. Por exemplo, tanto nas análises de dados da indústria quanto nas análises da academia, as linguagens abertas, incluindo Python e R, ganharam muita popularidade, uma vez que são de uso gratuito, aberto à modificações e disponibilizam para os pesquisadores compartilharem cada passo tomado para o processamento e análise de informações (GEIGER et al., 2018).

O desenvolvimento da manufatura aditiva segue este caminho, uma vez que existem diversos projetos gratuitos disponíveis na internet, com variadas especificações para atender uma maior gama de necessidades, ampliando a acessibilidade desta tecnologia à população. No Quadro 1 estão selecionadas algumas impressoras cujos projetos são abertos, estes equipamentos funcionam pelo processo FDM, diferenciando-se basicamente em sua estrutura.

A partir de análises dos modelos abaixo os membros do grupo PET EMB, selecionaram o projeto que melhor atendia às necessidades do grupo e que possuía maior acessibilidade de peças, o qual será detalhado posteriormente.

Quadro 1 - Impressoras FDM OpenProject

\begin{tabular}{|c|c|c|c|c|}
\hline Equipamento & Nome & $\begin{array}{c}\text { Preço } \\
\text { estimado } \\
(\mathbf{R} \$)\end{array}$ & $\begin{array}{c}\text { Volume de } \\
\text { Impressão } \\
(\mathbf{m m})\end{array}$ & $\begin{array}{c}\text { Dimensões da } \\
\text { Impressora } \\
\text { (mm) }\end{array}$ \\
\hline \\
\hline
\end{tabular}


(C) COBENGE

"Os desafios para formar hoje o engenheiro do amanhã"
$\mathrm{O} 1$ a $\mathrm{O} 3$ de dezembro

\section{Evento On-line}

\begin{tabular}{|c|c|c|c|c|}
\hline & $\begin{array}{c}\text { X3D XS } \\
\text { CoreXY 3D }\end{array}$ & $1.890,00$ & $190 \times 185 \times 140$ & $358 \times 341 \times 316$ \\
\hline & Printrbot Play & $2.150,00$ & $100 \times 100 \times 130$ & $355 \times 279 \times 304$ \\
\hline & Creality CR-10 & $2.150,00$ & $300 \times 300 \times 400$ & $540 \times 640 \times 310$ \\
\hline
\end{tabular}

Fonte: Produzido pelos autores

\section{MONTAGEM DA IMPRESSORA}

Para a montagem da impressora utilizou-se como base o projeto da RepRap, uma plataforma online, criada em 2005, com o intuito de desenvolver impressoras 3D mais baratas, acessíveis e que pudessem se autocopiar. A escolha por este projeto se deve pela facilidade e praticidade de se obter os componentes da estrutura, pois basicamente é composta de perfis de alumínio, porcas e parafusos.

Com as impressoras do Laboratório de Inovação e Desenvolvimento (LID) da Universidade Federal de Santa Catarina (UFSC) foram impressas as peças necessárias para o projeto. Durante o desenvolvimento foram realizadas algumas mudanças que resultaram em uma impressora do tipo CoreXY, na qual o extrusor se movimenta com a ajuda de um conjunto de correias, nos eixos x e y do plano cartesiano, e a plataforma no eixo z por meio de uma barra roscada. 
As peças foram encontradas na cidade o que facilitou a sua montagem, tantos os componentes estruturais como os eletrônicos são de fácil obtenção, contudo os custos envolvidos não são tão interessantes, pois adquirindo todos os componentes o seu custo ficou em torno de 2 mil reais, valor um pouco superior quando comparado a impressoras disponíveis para comprar em lojas. Na Figura 2, pode-se observar a impressora montada.

Figura 2 - Impressora montada

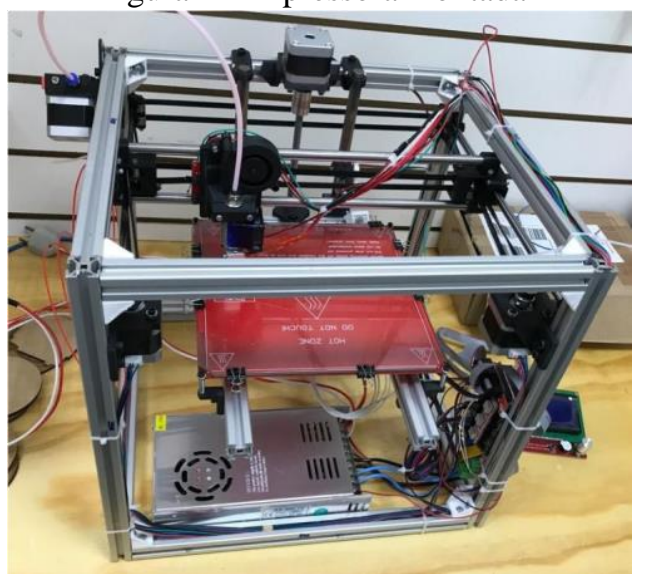

Fonte: Produzida pelos autores (2020)

No Quadro 2, encontram-se mais algumas especificações do modelo construído.

Quadro 2 - Especificações do modelo construído

\begin{tabular}{|c|c|c|c|}
\hline \multicolumn{4}{|c|}{ Especificações } \\
\hline $\begin{array}{c}\text { Largura } \\
\text { Profundidade } \\
\text { Altura }\end{array}$ & 380 & $\begin{array}{c}\text { Diâmetro do } \\
\text { bico extrusor } \\
\text { (mm) }\end{array}$ & 0,4 \\
\hline Custo (RS) & $2.000,00$ & $\begin{array}{c}\text { Temperatura } \\
\text { de trabalho } \\
\left({ }^{\circ} \mathbf{C}\right)\end{array}$ & $15^{\circ}$ a $40^{\circ}$ \\
\hline $\begin{array}{c}\text { Diâmetro do } \\
\text { filamento (mm) }\end{array}$ & 1,75 & $\begin{array}{c}\text { Software de } \\
\text { impressão }\end{array}$ & Simplify 3D \\
\hline $\begin{array}{c}\text { Área útil de } \\
\text { impressão (mm) } \\
\text { Largura }\end{array}$ & 200 & $\begin{array}{c}\text { Qualidade de } \\
\text { impressão, } \\
\text { altura da }\end{array}$ & 0,100 \\
Profundidade \\
Altura
\end{tabular}

Fonte: Produzida pelos Autores (2020) 
(C) COBENGE $2020=$

"Os desafios para formar hoje o engenheiro do amanhã"
01 a 03 de dezembro Evento On-line

Com a impressora construída foram realizados alguns testes, chegando à conclusão que o modelo construído tem uma boa repetibilidade e baixo erro nas dimensões circulares e lineares de uma peça, quando comparada a outros modelos comerciais (SILVA, 2019).

Atingimos o objetivo de montar o equipamento, no entanto passamos por algumas dificuldades, principalmente no que diz respeito às peças impressas, dentre as quais algumas possuíam falhas ou danificaram durante a construção.

\section{CONCLUSÃO}

Apesar de termos montado um modelo funcional e com baixo erro, o custo do projeto ultrapassou o estabelecido no orçamento inicial, portanto sendo mais viável a aquisição de uma impressora no mercado. Porém, a construção da impressora possibilitou que os membros do grupo PET do curso de Mecatrônica aprendessem mais sobre a tecnologia de manufatura aditiva, sobre pesquisa, desafios de trabalhar em equipe, bem como, conhecer e analisar diferentes projetos para um bom resultado.

Com este equipamento o grupo PET poderá realizar projetos que necessitam de protótipos, também poderá disponibilizar para outros estudantes do campus a impressora para a realização de diversos projetos. Um exemplo é o jogo da memória de alto relevo para pessoas com deficiência visual, desenvolvido por membros do Laboratório de Inovação e Desenvolvimento (LID), outro é o trabalho da disciplina de Representação Gráfica, no qual o professor pede que cada aluno desenvolva uma peça específica, muitos optam por fazer via manufatura aditiva.

Figura 3 - Jogo da Memória

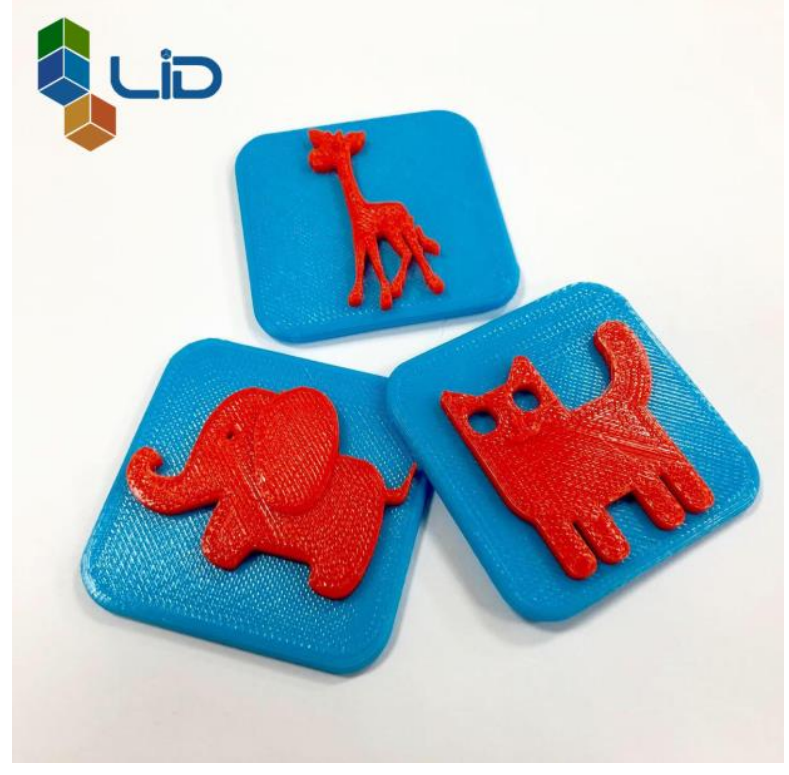

Fonte: LID 
As experiências adquiridas durante a montagem da impressora constituem-se como referências para o desenvolvimento de outros projetos acadêmicos ou profissionais dos membros do PET EMB envolvidos.

\section{Agradecimentos}

Os autores agradecem o Ministério da Educação e ao FNDE pela oportunidade de bolsa dentro do Programa de Educação Tutorial das Engenharias da Mobilidade (PET EMB). À universidade pelo apoio ao programa e apoio ao nosso projeto. Ao nosso tutor pelo suporte em todo projeto, e o suporte e trabalho conjunto dos colegas de equipe.

\section{REFERÊNCIAS}

AGARWALA, M. et al. Direct selective laser sintering of metals. Rapid Prototyping Journal, v.1 n.1, p.26-36, 1995.

EVANS, Brian. Practical 3D Printers: The Science and Art of 3D Printing. 1a edição, Apress, 2012.

GEIGER, R. S. et al. The types, roles, and practices of documentation in data analytics open source software libraries. Computer Supported Cooperative Work (CSCW), v.27, n.3-6, p.767-802, 2018.

GRAMES, E. What exactly is FDM 3D printing? We simply explain how it works as well as cover the advantages and disadvantages. Disponível em: https://all3dp.com/2/fused-deposition-modeling-fdm-3d-printing-simply-explained/.

Acesso em: 26 de maio de 2020

JABBOUR, A. et al. Análise da relação entre manufatura enxuta e desempenho operacional de empresas do setor automotivo no Brasil. Revista de Administração (São Paulo), São Paulo, v.48 n.4, 2013.

KODAMA, H. Automatic method for fabricating a three-dimensional plastic model with photohardening polymer. Review of Scientific Instruments, v.52, n.11, p.1770-1773, 1981.

LID. Jogo da memória para crianças com deficiência visual. Disponível em: https://lid.joinville.ufsc.br/projetos/jogo-da-memoria/. Acesso em: 20 de setembro de 2020.

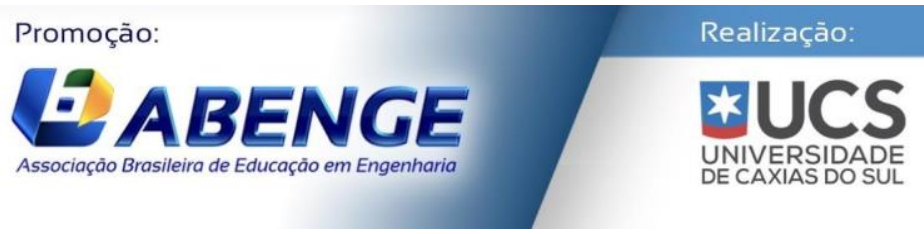


MASOOD, S. Intelligent rapid prototyping with fused deposition modelling. Rapid

Prototyping Journal, v.2 n.1, p. 24-33, 1996.

PANNETT, Len. Supercharg3d: How 3D Printing Will Drive Your Supply Chain. $1^{\text {a }}$ edição, New Jersey: John Wiley \& Sons, 2019.

PAOLETTI, Ingrid; CECCON, Lorenzo. The Evolution of 3D Printing in AEC: From Experimental to Consolidated Techniques. IntechOpen, 2018.

RAULINO, B. R.; Manufatura Aditiva: Desenvolvimento de uma Máquina de Prototipagem Rápida Baseada na Tecnologia FDM (Modelagem por Fusão e Deposição). Trabalho de Graduação em Engenharia de Controle e Automação, Publicação FT.TG-nº, Faculdade de Tecnologia, Universidade de Brasília, Brasília, DF, 141p, 2011.

REDWOOD, Ben; SCHÖFFER, Filemon; GARRET, Brian. The 3D Printing Handbook: Technologies, design and applications. Amsterdam: 3D Hubs, 2017.

RENAP, K.; KRUTH, J. P. Recoating issues in stereolithography. Rapid Prototyping Journal, v.1 n.3, p. 4-16, 1995.

SILVA, B. A.; Avaliação de erros dimensionais de uma peça padrão em diferentes máquinas geradas pelo processo de modelagem por deposição fundida. Trabalho de Conclusão de Curso em Bacharelado em Ciência e Tecnologia, Universidade Federal de Santa Catarina, Joinville, SC, 2019.

SHAH, R.; WARD, P. T. Lean manufacturing: context, practice bundles, and performance. Journal of Operations Management, v.21, n.2, p.129-149, 2003.

STEENBEEK, J. et al. Ecopath with Ecosim as a model-building toolbox: source code capabilities, extensions, and variations. Ecological Modelling, v.319, p.178-189, 2015.

VOLPATO, Neri et al. Prototipagem Rápida: Tecnologias e Aplicações. $1^{\text {a }}$ ed. Editora Blucher, 2007. 


\title{
EVALUATION AND VIABILITY OF LOW-COST 3D PRINTERS FOR ACADEMIC ACTIVITIES
}

\begin{abstract}
This work was carried out by members of the Tutorial Education Program for Mobility Engineering (PET EMB) on the Joinville campus of the Federal University of Santa Catarina (UFSC). It is an experience report that aims to analyze different additive manufacturing projects and also describe as members experiences in building a low-cost open design model of printing equipment by the FDM - Fused Deposit Modeling process. During the construction of the project, challenges, difficulties and learning were encountered, constituting an experience that contributed to the training of the members involved.
\end{abstract}

Keywords: Additive Manufactory. Open Projects. FDM. Low cost. PET. 